\title{
Thermal conductivity of advanced architected cellular materials
}

\author{
A. Mirabolghasemi \\ Department of Bioresource Engineering \\ McGill University \\ Montreal, Canada \\ D. Therriault \\ Department of Mechanical Engineering \\ Polytechnique Montreal \\ Montreal, Canada
}

\begin{abstract}
Architected cellular materials, as a novel class of low density materials, gain their unprecedented multifunctional performance mainly from their underlying architecture. In this paper, we focus on thermal conductivity of cellular materials. Standard mechanics homogenization with periodic boundary conditions is used to determine the thermal conductivity of cells with supershape pores. The computational results confirm that a wide range of possible anisotropic behaviour for thermal conductivity is achievable for cellular materials. Effective thermal conductivity of shellular materials based on three triply periodic minimal surfaces are also compared with those of cells with supershape pores. It is found that unlike the shellular materials, which only cover a narrow portion of thermal conductivity vs. relative density chart, cellular materials with anisotropic effective thermal conductivity could be engineered by employing supershape pores in cells.
\end{abstract}

Keywords - Architected cellular materials; Thermal conductivity; Homogenization; Supershape pore; Shellular materials

\section{INTRODUCTION}

Limited material and energy resources, economical restrictions, and concerns over the prospect of global climate changes, promote the design and manufacturing of lightweight materials with tunable multifunctional properties. Architected cellular materials are one of the cutting-edge alternative lightweight and optimized materials which can simultaneously satisfy multiple functionalities, from structural stiffness to thermal insulation and heat exchanging [1].

Cellular materials are divided into two categories: foams, where a gaseous phase is randomly dispersed in a continuous solid medium, and periodic lattices, which consist of a periodic architected cell [2]. The concept of using cellular metals emerged in early 1970s, by inspiration from the excellent

\author{
A. H. Akbarzadeh \\ Department of Bioresource Engineering \& \\ Department of Mechanical Engineering \\ McGill University \\ Montreal, Canada \\ Email: hamid.akbarzaeh@mcgill.ca
}

\author{
D. Rodrigue \\ Department of Chemical Engineering \\ Université Laval \\ Quebec, Canada
}

properties of natural materials like bone, wood and cork. Hexagonal honeycomb structures in sandwich cores, used in aviation industry, can be named as one of the first applications of periodic cellular materials [3]. Driven by the high performance of these cellular materials and recent development of additive manufacturing, advanced polymeric and metallic cellular materials are being widely fabricated. A non-exhaustive list of applications of cellular materials found in the literature includes: lightweight structural elements in aircrafts and highspeed trains, energy-absorbing elements in automotive industry, thermal insulation, thermal energy storage devices, hydrogen storage tanks, and scaffold for tissue engineering [2-11].

To exploit multifunctional potentials of cellular materials, a mathematical predictive model, capable of accurately predicting their effective properties, is of crucial importance. Early efforts on this subject range from the simple volumetric averaging of properties of constituent cellular materials, so-called as 'rule of mixtures method', to several empirical equations for predicting the multiphysical properties of cellular materials [2, 12]. Investigations have clearly shown that cell architecture, in addition to the properties of the constituents' materials, plays a significant role in the emergence of their outstanding properties $[13,14]$. In this regards, as thermal conductivity, electrical conductivity and magnetic permeability are all mathematically described by the Laplace equation, existing approaches used in the electric and magnetic fields have been also applied for thermal properties of cellular materials $[15,16]$. The introduction of thermal-circuit method, based on the analogy between the electrical and thermal conductivities, could be considered as a turning point in theoretical modelling of thermal conductivity of cellular materials [16]. It is worth mentioning that most of the analytical models have been mainly developed for random pore distribution [17], or simple pore topologies, e.g. cubic, circular, cylindrical, and spherical [18, 19], leaving more complex periodic architectures with potential superior multifunctional properties unexplored. Although analytical upper and lower bounds, e.g. those bounds presented by Hashin 
and Strikman [20] or thermal-circuit method, are valuable for estimating the effective thermal conductivity with a minimum knowledge about the actual heat flow and temperature profile in cellular materials, advanced computational models, e.g. Latticebased Monte Carlo approach [21], micropoloar modelling [22], and standard mechanics [23] and asymptotic homogenizations [24], have been developed to precisely take into account the effect of microarchitecture of cellular materials to accurately predict their effective thermal properties. The physical properties and application of cellular materials have been summarize in several textbooks, among which the book by Gibson and Ashby [2] provide the most comprehensive overview.

In this paper, we present two methodologies for predicting the effective thermal conductivity of architected cellular materials: (1) Theoretical modelling using the thermal-circuit method [25] and (2) Computational modeling based on standard mechanics homogenization. While the theoretical model is used to provide a narrow bound for thermal conductivity of cellular materials with simple 2D microarchitecture, standard mechanics homogenization is used to determine the exact thermal conductivity of cellular materials with complex 2D microarchitecture. To highlight the effect of cell topology on the thermal conductivity of periodic cellular materials, modified supershape formula [26] has been employed to create 2D pore shapes on square cells. Moreover, to address the ever growing interests on the lightweight 3D cellular materials, shellular materials based on three triply-periodic minimum surfaces have been analyzed and compared with the rest of selected cellularbased metamaterials.

\section{CAD DESIGN OF ARCHITECTED CELlular MATERIALS}

To apply computational standard mechanics homogenization to explore the effect of topology and relative density on the effective thermal conductivity of cellular materials, 2D square unit cell with one supershape pore and 3D cubic shellular representative volume element (RVE) are selected.

\section{A. 2D RVES}

As a powerful formula for creating a variety of pore shapes, superformula (1) is selected and modified to generate void geometries while scaling and rotation $(\beta)$ parameters are considered to increase the possibilities of pore topologies.

$$
\begin{array}{r}
{\left[\begin{array}{l}
\mathrm{x} \\
\mathrm{y}
\end{array}\right]=[|\cos (\mathrm{m} \phi / 4)|+|\sin (\mathrm{m} \phi / 4)|]^{\mathrm{n}}\left[\begin{array}{l}
\cos (\phi) \\
\sin (\phi)
\end{array}\right]} \\
(-\pi \leq \phi \leq \pi, \mathrm{m}=1 \sim 8, n=-5 \sim 5)
\end{array}
$$

Fig 1 shows sample 2D RVEs for different topological parameters. The pore shape corresponding to each set of geometrical parameters, introduced in (1), is directly modeled inside ANSYS APDL (Fig. 2) by creating several keypoints and using them to make spline curves. The area surrounded by the spline curves is formed, scaled, rotated and moved to the center of the RVE to be subtracted from the RVE and to finalize the 2D RVE.

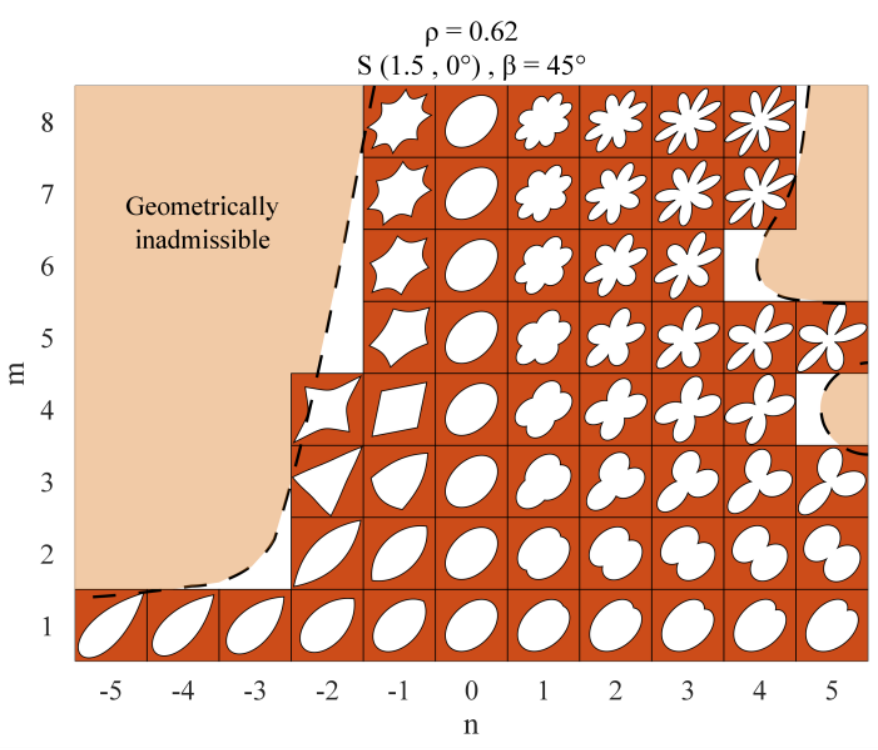

Figure 1. Sample 2D RVEs for different parameters: $\rho=0.62$, scaled by 1.5 in horizontal direction, and rotated by $45^{\circ}$

\section{B. 3D shellular RVEs}

A Triply Periodic Minimal Surface (TPMS) is a non-selfintersecting surface in $\mathrm{R}^{3}$, which has a crystalline structure repeated in three independent directions and has zero mean curvature at each point [27]. The presence of TPMS in natural structures, like biological membranes [28] and crystals [27], has inspired researchers to consider TPMS architectures in tissue engineering, and biomimetic material design [29-32]. It is worth mentioning that "Shellular" term has recently been used in the literature to represent thin TPMS cellular shells. Among many known TPMSs, Schwarz's Primitive (P) and Diamond (D) and Schoen's Gyroid (G) are selected. These surfaces can be trigonometrically approximated using the following level surface equations [33]:

$$
\begin{array}{llr}
\text { G: } & \cos x \cdot \sin y+\cos y \cdot \sin z+\cos z \cdot \sin x=f \quad(f=0) \\
P \cdot & \cos x+\cos y+\cos z=f \quad(f=0,0 \cdot 4,0.8) \\
\text { D: } & \begin{array}{l}
\sin x \cdot \sin y \cdot \sin z+\sin x \cdot \cos y \cdot \cos z+ \\
\\
\cos x \cdot \sin y \cdot \cos z+\cos x \cdot \cos y \cdot \sin z=f
\end{array} \quad(f=0)
\end{array}
$$

We develop a MATLAB code to solve the level surface equations to determine the coordinates of several points on the surface. These points together with multiple cross section curves satisfying the surface equations are firstly created inside Solidworks using a Visual Basic code and then are used to create a smooth surface. This part of the surface is then patterned to create the mid-surface of the shellular RVE and subsequently thickened in order to add the desired thickness (as illustrated in Fig. 3). To focus more on the lightweight structures, five thicknesses $\left(t_{\text {rel }}\right)$ between 2 to 10 percent of RVE's length are considered. Fig. 4 demonstrates some of the selected shellular architectures. 

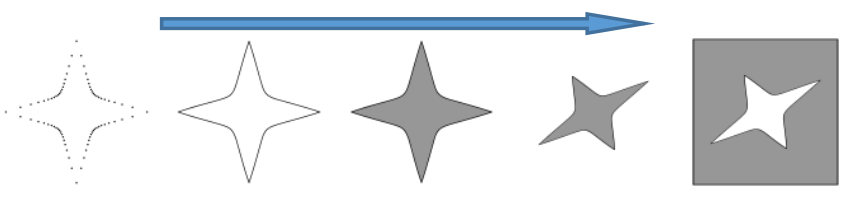

Figure 2. Different steps in modeling of 2D RVEs

\section{PREDICTIVE METHODS}

Fourier's law assumes the following linear relation between heat flux $(\overrightarrow{\mathrm{q}})$ and temperature gradient $(\vec{\nabla} \mathrm{T})$ through a symmetric thermal conductivity tensor $\left(\mathrm{K}_{\mathrm{eff}}\right)$ :

$$
\vec{q}=-K_{e f f} \vec{\nabla} T
$$

For a thermally isotropic homogenous solid material, $K_{\text {eff }}$ tensor reduces to $\mathrm{KI}$, where $\mathrm{I}$ is the identity tensor and $\mathrm{K}$ is called isotropic thermal conductivity. However, in the case of a cellular material, overall thermal conductivity is generally anisotropic and depends on microarchitectural parameters of the cells [1].

Since the simplistic volumetric averaging approach is indifferent to cell architecture, it is unable to capture the anisotropic thermal properties of cellular materials and its prediction can significantly overestimate the effective thermal conductivity. To address these shortcomings, several analytical and computational methods have been developed, among which thermal-circuit method (or Resistor approach) has been widely used for the theoretical upper and lower bounds of thermal conductivity for a given cell geometry [4]. More advanced methods, e.g. computational homogenization, are required to exactly model the microarchitecture of cellular materials.

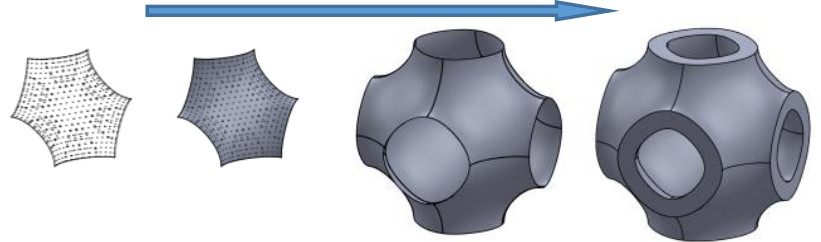

Figure 3. Steps for 3D modeling of a 3D shellular RVE

It is worth mentioning that the contribution of heat transfer mechanisms other than conduction, i.e. radiation and convection, is assumed to be small and consequently disregarded. For a cellular material made by a highly conductive matrix and an empty void or a gaseous inclusion, this assumption is valid for small pore sizes working at temperature ranges close to the ambient [34-37].

\section{A. Analytical model}

Using the analogy between thermal and electric fields, the thermal circuit method with parallel and series configurations is used to derive closed-from expressions for the upper and lower bounds of thermal conductivity of cellular materials with rectangular or elliptic inclusion/pore. In this approach, thermal gradient is analogous to electric voltage, the heat flow represents electric current and thermal resistance, being equal to the reciprocal of thermal conductivity for a unit cell, corresponds to electric resistance [25]. To establish this model, as presented in Fig. 5, the unit cell is divided into rectangular elements acting as thermal resistors, while the heat flux is considered to be along " $y$ " direction and perfect thermal contact is assumed between the matrix (solid cell walls) and the filler material (such as air for cellular materials).

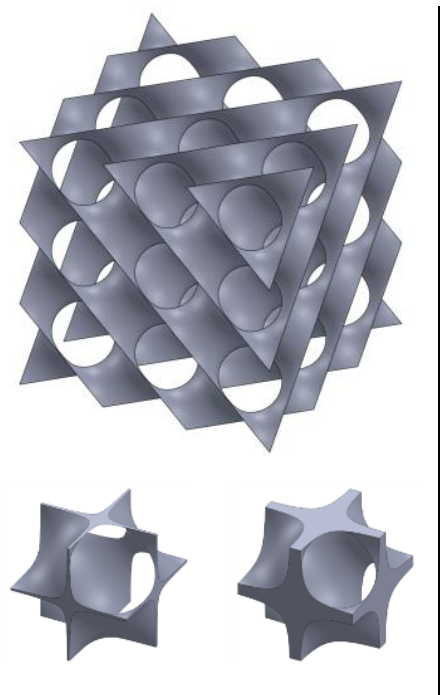

(a)

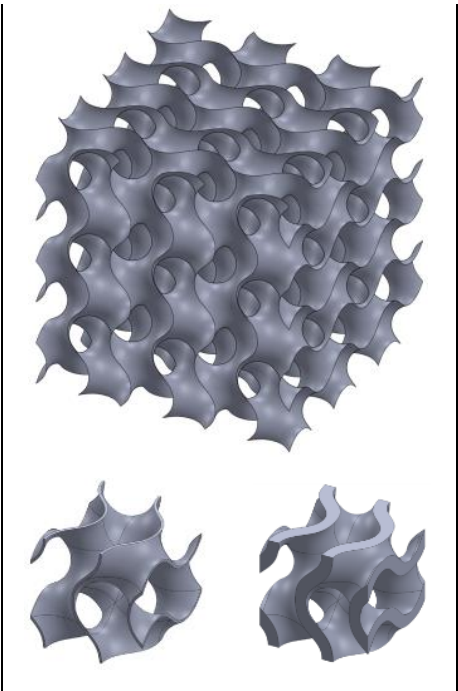

(b)

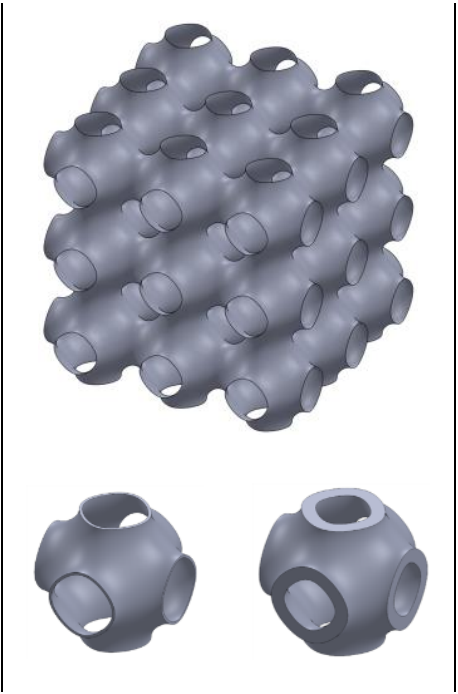

(c)
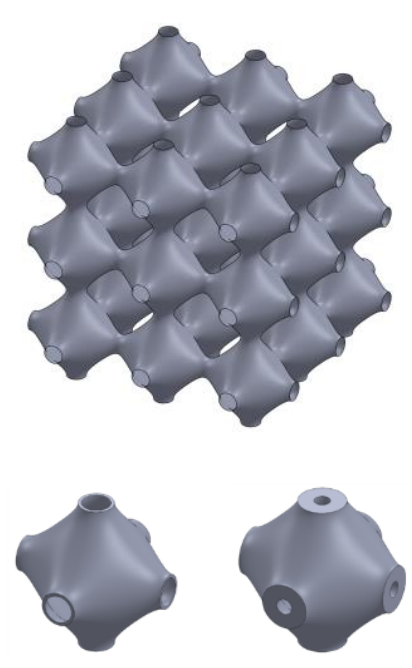

(d)

Figure 4. $3 \times 3 \times 3$ TPMS cells together with two shellular RVEs at 0.02 and 0.10 relative thicknesses: (a) Schwarz D, (b) Gyroid, (c) Schwarz P with $f=0$, and (d) Schwarz $P$ with $f=0.8$ 


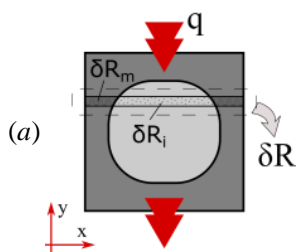

$$
\begin{gathered}
\delta R^{y}=\left(\frac{1}{\delta R_{m}^{y}}+\frac{1}{\delta R_{i}^{y}}\right)^{-1} \\
R^{y}=\sum \delta R^{y}
\end{gathered}
$$

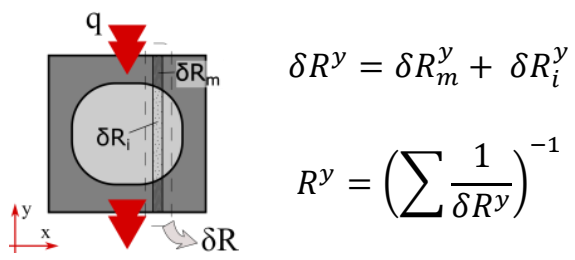

Figure 5. Schematic view of the thermal resistant elements for: (a) Horizontal iso-thermal lines and (b) Vertical adiabatic lines

Closed-form thermal conductivity formulations are presented in Table I, where $k_{m}$ and $k_{i}$ are the thermal conductivity of the matrix and inclusion respectively, $\lambda_{a}=a / l \quad, \quad \lambda_{b}=b / l \quad, \quad \lambda_{k}=k_{i} / k_{m}(<1)$, $\theta=\operatorname{atan}\left(\sqrt{\left(\left(1-\lambda_{k}\right) \lambda_{a}\right)^{-2}-1}\right.$ and $A=\lambda_{b}\left(1 / \lambda_{k}-1\right)$. It can be found that 'Vertical adiabatic lines' expressions for both rectangular and elliptic geometries reduce to $k_{e f f}^{y y} / k_{m}=1-\lambda_{a}$ when $k_{i}=0$, which corresponds to cellular materials with empty pores. The relative density of the matrix can be calculated as $\left(\rho_{m}\right)_{r e l}=\left(l^{2}-a b\right) / l^{2}=1-\lambda_{a} \lambda_{b}$ and $\left(\rho_{m}\right)_{\text {rel }}=\left(l^{2}-\pi a b / 4\right) / l^{2}=1-\pi \lambda_{a} \lambda_{b} / 4$, for cells with rectangular and elliptic inclusion/pore, respectively. As the symmetry in the selected architectures of matrix and inclusion/pore dictates, the off-diagonal terms of the thermal conductivity tensor are zero $\left(K_{\text {eff }}^{x y}=0\right)$ and thermal conductivity in " $x$ " and " $y$ " are equal.

\section{B. Computational standard mechanics homogenization}

Under the assumption that an RVE of a periodic cellular material is repeated in all three directions and the RVE is far from the boundaries, the following periodic boundary conditions (4) together with independent unit thermal gradients (5) on the cell boundaries are adopted [23]:
Periodicity in $x$-direction:

$$
T\left(x_{0}, y, z\right)-T\left(x_{0}+l_{x}, y, z\right)=l_{x}\left(\frac{\partial T}{\partial x}\right)_{i}
$$

Periodicity in $y$-direction:

$$
T\left(x, y_{0}, z\right)-T\left(x, y_{0}+l_{y}, z\right)=l_{y}\left(\frac{\partial T}{\partial y}\right)_{i}
$$

Periodicity in z-direction:

$$
T\left(x, y, z_{0}\right)-T\left(x, y, z_{0}+l_{z}\right)=l_{z}\left(\frac{\partial T}{\partial z}\right)_{i}
$$

Independent unit thermal gradients:

$$
(\overline{\nabla T})_{i}=\left(\frac{\partial T}{\partial x}, \frac{\overline{\partial T}}{\partial y}, \overline{\partial T}\right)_{i z}= \begin{cases}(1,0,0), & i=1 \\ (0,1,0), & i=2 \\ (0,0,1), & i=3\end{cases}
$$

where $i$ is the thermal loading case number and $\mathrm{x}_{0}, \mathrm{y}_{0}$ and $\mathrm{z}_{0}$ are the locations of the three faces of the cubic RVE, while $\mathrm{l}_{\mathrm{x}}, \mathrm{l}_{\mathrm{y}}$ and $\mathrm{l}_{\mathrm{z}}$ represent RVE's dimensions along " $x$ ", " $y$ " and " $z$ " axis, and $\overline{\nabla T}$ is the average macroscopic thermal gradient applied to the RVE's boundaries in order to calculate microscopic thermal gradients inside the cell. Since the RVE is

TABLE I. CLOSED-FORM EXPRESSIONS FOR $k_{e f f}^{y y} / k_{m}$ OF CELLS MADE BY THERMALLY ISOTROPIC AND HOMOGENEOUS MATRIX AND FILLER MATERIALS, HAVING RECTANGULAR AND ELLIPTIC INCLUSION/PORE SHAPES, UNDER THE ASSUMPTIONS OF 'HORIZONTAL ISO-THERMAL LINES' OR 'VERTICAL ADIABATIC LINES'

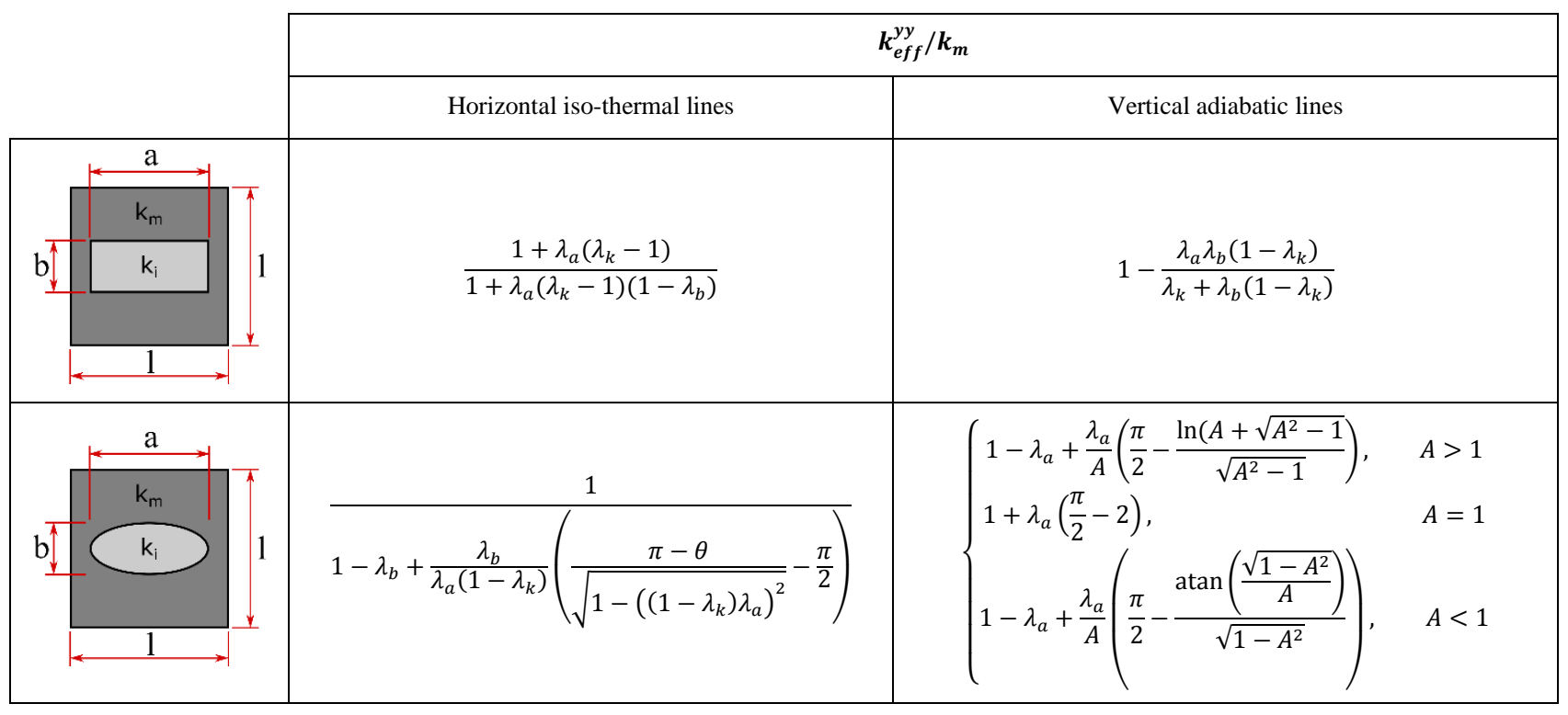


a unit square in two-dimensional (2D) analysis and a unit cube in three-dimensional (3D) analysis of this research, Eqs. (4) and (5) can be further simplified to:

$$
\left(\begin{array}{l}
\mathrm{T}\left(\mathrm{x}_{0}, \mathrm{y}, \mathrm{z}\right)-\mathrm{T}\left(\mathrm{x}_{0}+1, \mathrm{y}, \mathrm{z}\right) \\
\mathrm{T}\left(\mathrm{x}, \mathrm{y}_{0}, \mathrm{z}\right)-\mathrm{T}\left(\mathrm{x}, \mathrm{y}_{0}+1, \mathrm{z}\right) \\
\mathrm{T}\left(\mathrm{x}, \mathrm{y}, \mathrm{z}_{0}\right)-\mathrm{T}\left(\mathrm{x}, \mathrm{y}, \mathrm{z}_{0}+1\right)
\end{array}\right)_{\mathrm{i}}=\left(\begin{array}{l}
1 \\
0 \\
0
\end{array}\right)_{\mathrm{i}=1},\left(\begin{array}{l}
0 \\
1 \\
0
\end{array}\right)_{\mathrm{i}=2},\left(\begin{array}{l}
0 \\
0 \\
1
\end{array}\right)_{\mathrm{i}=3}
$$

In 2D analysis, " $z$ " dimension diminishes leading to two independent thermal loading cases. Energy balance equation, i.e. energy equation combined with Fourier heat conduction over the $\mathrm{RVE}$, is solved using finite element method (FEM). The effective thermal conductivity of the cell can then be calculated by the volumetric averaging of the resultant heat flux based on standard mechanics homogenization:

$$
\bar{K}_{i j}=\frac{1}{V_{R V E}} \int K_{i k} M_{k j}^{T} d V_{R V E} \quad(i, j, k=1,2,3)
$$

where $\overline{\mathrm{K}}_{\mathrm{ij}}$ is the effective thermal conductivity tensor, $\mathrm{V}_{\mathrm{RVE}}$ represents the volume of the RVE, $K_{i k}$ is the local thermal conductivity tensor, and the matrix of $\mathrm{M}^{\mathrm{T}}$ relates the average and the local temperature gradients by $\nabla T=M^{T} \overline{\nabla T}$.

\section{RESULTS AND DISCUSSION}

To show the effect of filler material on the overall thermal conductivity and to examine the validity of neglecting the thermal conductivity of the gas inside the cellular materials, the homogenized thermal conductivity of a square cell with an empty square pore is benchmarked against the effective thermal conductivity of the same cell infilled with air at room temperature. As shown in Fig. 6, even for relative densities as low as $10 \%$, air's thermal conductivity can be neglected without affecting the effective thermal conductivity when the thermal conductivity of the matrix material is several orders of magnitude higher than the air, such as in metallic cellular materials.

Under the validated assumption of neglecting the air, derived closed form expression for the cells with rectangular and elliptical pore topologies are compared with the results obtained by standard mechanics homogenization. As illustrated in Fig. 7, for all aspect ratios of pores $(1,2$ and 3 in the horizontal direction), distances between higher and lower bounds of the effective thermal conductivity of the cells with elliptical pores are larger than those for the cells with rectangular pores. Moreover, by increasing the relative density, the gap between the theoretical bounds increases. In this case, although the pore geometry is accounted for deriving the theoretical upper and lower bounds, the simplifying assumptions made in deriving the closed form formula based on the thermal-circuit method make it impossible to accurately predict the effective thermal conductivity.

Close to the smallest possible relative density for each aspect ratio of pore, the effective thermal conductivity of cells with elliptical pores reveals sharper drops in comparison with cells with rectangular pores. This is partly because of the higher rate of decreasing of the minimum wall thickness with respect to decreasing in the relative density for the former topologies.

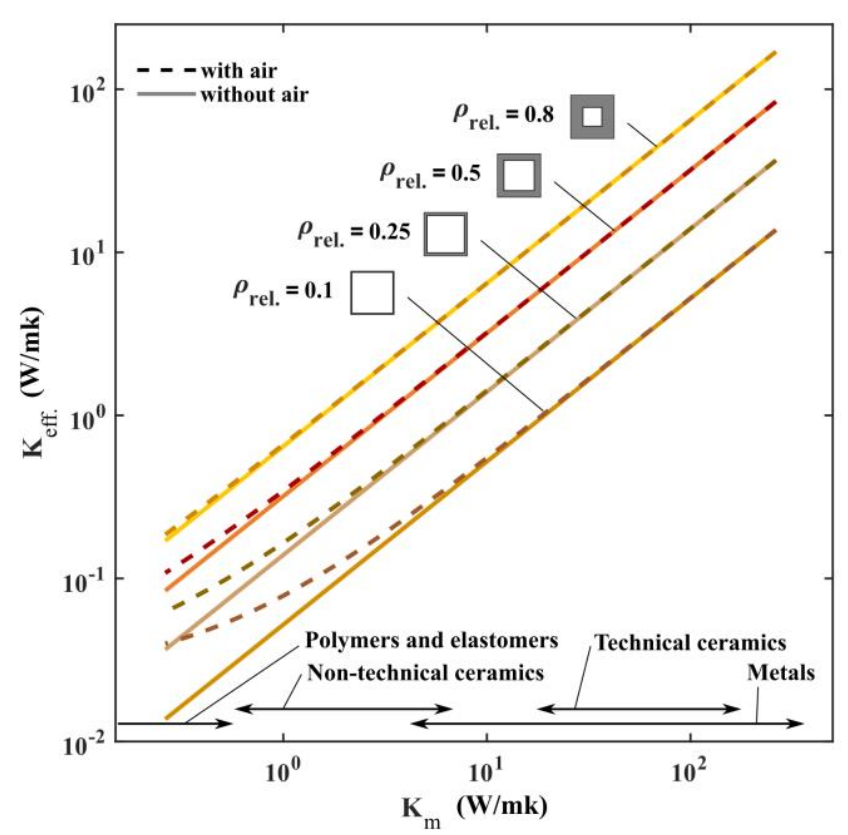

Figure 6. Effective thermal conductivity of air-filled ( $k_{\text {air }}=0.0263 \mathrm{~W} / \mathrm{m} . \mathrm{K}$ ) and empty $\left(k_{i}=0\right)$ cellular materials for a square pore shape versus thermal conductivity of the solid matrix (plotted in a log-log scale)

The standard mechanics homogenization is applied to cells with supershape pore topologies, while different aspect ratios (1 to 3 , with 0.5 increments) and rotations (generally from 0 to $90^{\circ}$ ) of the pore has been taken into account. The predicted in-plane effective thermal conductivities are generally anisotropic, therefore instead of $K_{x x}, K_{y y}$ and $K_{x y}$, the effective principal thermal conductivities are presented in Fig. 8. Sample relative densities are highlighted with different colors to show the achievable range of $K_{I}$ and $K_{2}$ by the chosen pore topologies. The large area of $K_{l}$ versus $K_{2}$ for each relative density allows the engineering of architected cellular materials, while keeping the weight constant.

Unlike most of the considered 2D pore geometries, the computational analysis shows that $\mathrm{P}, \mathrm{D}$ and $\mathrm{G}$ types of shellular materials are thermally isotropic and thus only one value is reported in Fig. 9 as the thermal conductivity for each microarchitecture. The G, D and P shellular materials (when $f=$ 0 ) are found to have almost equal thermal conductivity at each relative density, which is in agreement with the findings in [38]; however, by increasing the value of $f$ for the $\mathrm{P}$ shellular materials, the effective thermal conductivity decreases.

\section{CONCLUDING REMARKS}

Theoretical and computational methodologies have been introduced in this paper for obtaining the effective thermal conductivity of architected cellular materials. We first examine the validity of neglecting the thermal conductivity of the air inside the pores of cellular materials when we determine the effective thermal conductivity of cellular materials. We then derive closed-form expressions for the upper and lower bounds of the in-plane effective thermal conductivity of cellular materials with rectangular and elliptic pores. The computational predictions of standard mechanics homogenization are also used 


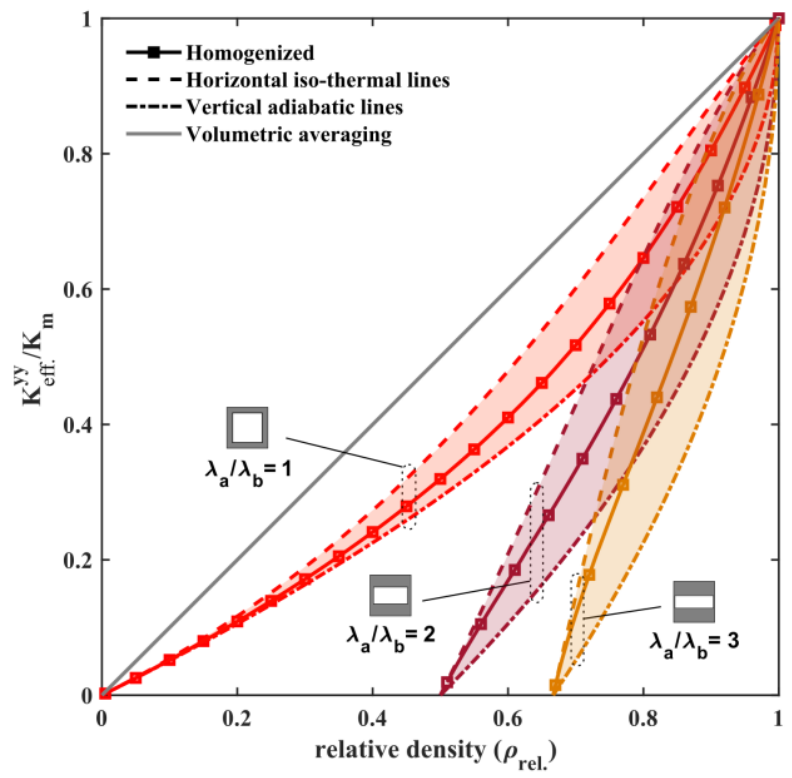

(a)

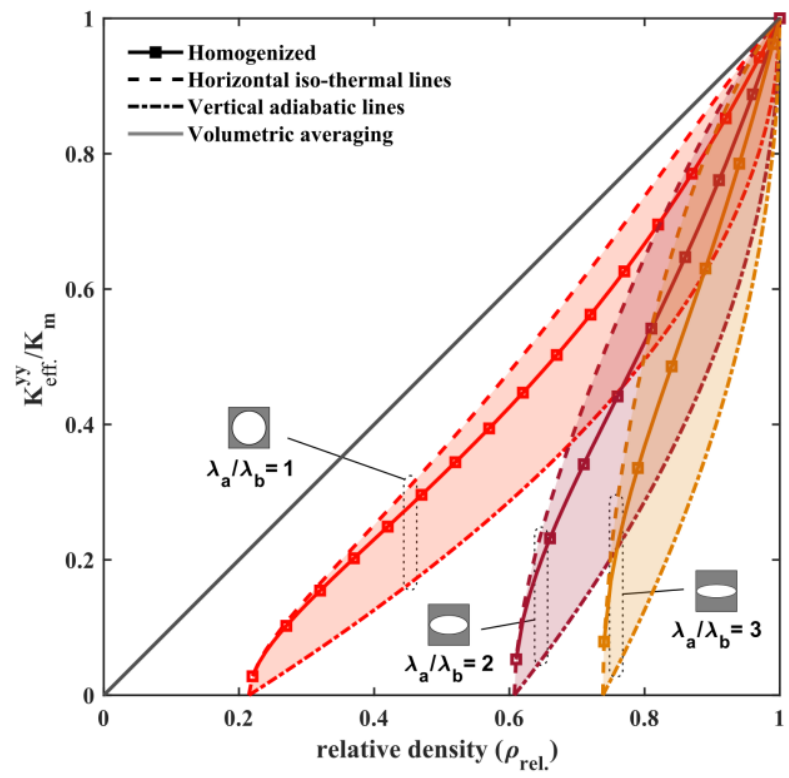

(b)

Figure 7. Theoretical upper and lower bounds along with computational homogenized values of effective thermal conductivity in " $y$ " direction, normalized by the thermal conductivity of the solid matrix: (a) Rectangular pore and (b) elliptic pore

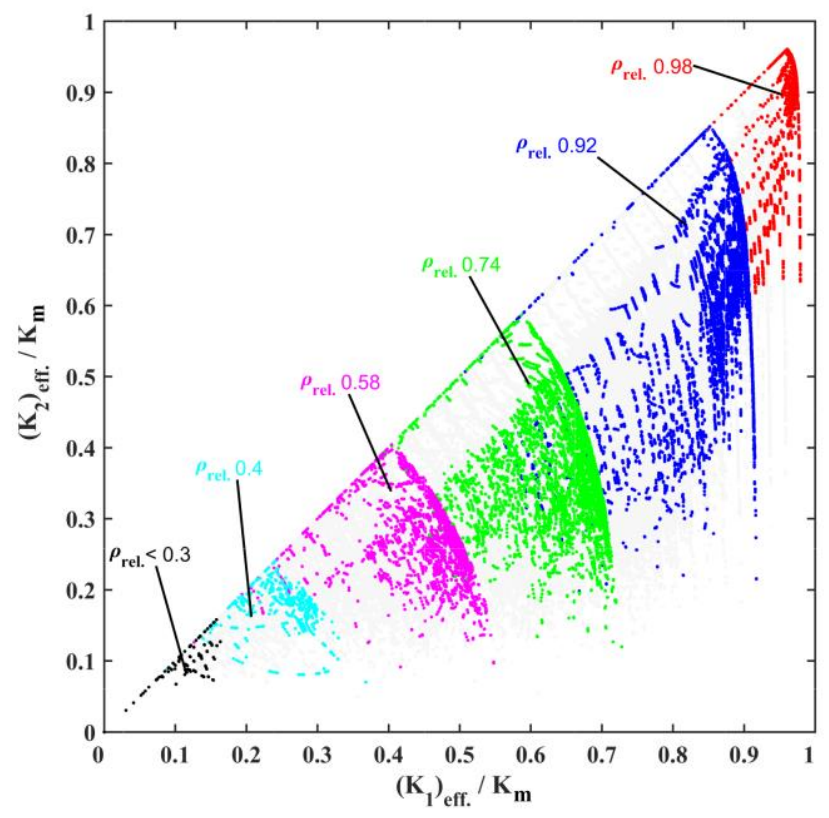

Figure 8. Normalized effective principal thermal conductivities, obtained using standard mechanics homogenization (for 2D supershape voids)

as benchmark for analyzing the effect of cell microarchitecture on effective thermal conductivity. The predictions show that increasing aspect ratio of the pores, widens the gap between the theoretical upper and lower bounds of thermal conductivity. Computational homogenized predictions are found to be close to the theoretical upper bound for small relative densities. The results present a wide range of achievable anisotropic effective thermal conductivity for different relative densities. Different TPMSs are also selected to create 3D shellular materials. For the considered range of relative density $\left(\rho_{\text {rel }}<25 \%\right), \mathrm{G}, \mathrm{D}$ and P

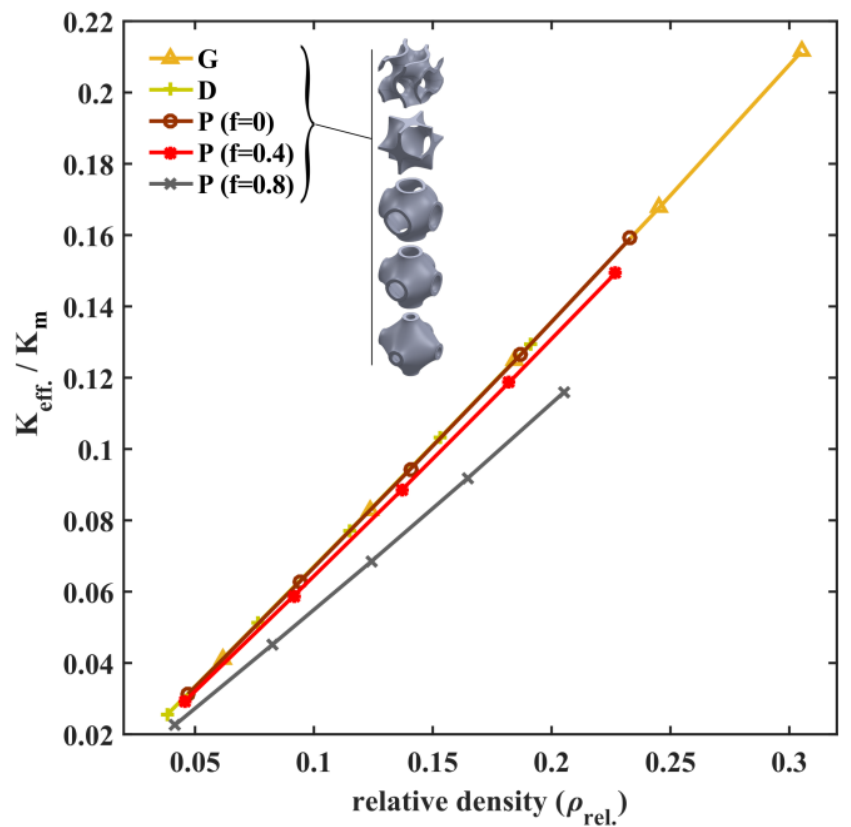

Figure 9. Effective thermal conductivity of shellular materials, normalized by thermal conductivity of the solid matrix

shellular materials with $f=0$, are found to have equal homogenized thermal conductivity. The methodology presented in this paper sheds light on the thermal application of lightweight and mechanically robust advanced cellular materials manufacturable by additive manufacturing technology.

\section{ACKNOWLEDGMENT}

A.H. Akbarzadeh acknowledges the financial support by McGill University and Natural Sciences and Engineering 
Research Council of Canada (NSERC) through NSERC Discovery Grant RGPIN-2016- 29 0471. The authors also acknowledge financial support by the Research Center for High Performance Polymer and Composite Systems (CREPEC).

\section{REFERENCES}

1. Akbarzadeh Shafaroudi, A. and S. Rankohi, Multifunctional and Multiphysics Materials As Load-. 2016: p. 1-9.

2. L.J. Gibson and M.F. Ashby, Cellular solids: Structure \& properties, Oxford: Pergamon Press, ISBN: 0-08-036607-4, 1988, $357+$ ix pages, \$35.00. Advances in Polymer Technology, 1989. 9(2): p. 165-166.

3. Öchsner, A. and T. Fiedler, Effective Thermal Properties of HollowSphere Structures: A Finite Element Approach, in Cellular and Porous Materials. 2008, Wiley-VCH Verlag GmbH \& Co. KGaA. p. 31-71.

4. Baillis, D. and R.m. Coquard, Radiative and Conductive Thermal Properties of Foams. Cellular and Porous Materials: Thermal Properties Simulation and Prediction, 2008: p. 343-384.

5. Belova, I.V. and G.E. Murch, Thermal Properties of Composite Materials and Porous Media: Lattice-Based Monte Carlo Approaches, in Cellular and Porous Materials. 2008, Wiley-VCH Verlag GmbH \& Co. KGaA. p. 73-95.

6. Belova, I.V., et al., Analysis of anisotropic behaviour of thermal conductivity in cellular metals. Scripta Materialia, 2011. 65: p. 436439.

7. Fiedler, T., et al., Determination of the thermal conductivity of periodic APM foam models. International Journal of Heat and Mass Transfer, 2014. 73: p. 826-833.

8. Gasia, J., L. Miró, and L.F. Cabeza, Materials and system requirements of high temperature thermal energy storage systems: A review. Part 2: Thermal conductivity enhancement techniques. Renewable and Sustainable Energy Reviews, 2016. 60: p. 15841601.

9. Kumar, P., F. Topin, and J. Vicente, Determination of effective thermal conductivity from geometrical properties: Application to open cell foams. International Journal of Thermal Sciences, 2014. 81: p. 13-28.

10. Montazerian, H., et al., Porous scaffold internal architecture design based on minimal surfaces: A compromise between permeability and elastic properties. Materials and Design, 2017. 126: p. 98-114.

11. Wang, W., et al., Analytical design of effective thermal conductivity for fluid-saturated prismatic cellular metal honeycombs. Theoretical and Applied Mechanics Letters, 2016. 6: p. 69-75.

12. Ranut, P., On the effective thermal conductivity of aluminum metal foams: Review and improvement of the available empirical and analytical models. Applied Thermal Engineering, 2016. 101: p. 496524.

13. Akbarzadeh, A.H., et al., Electrically conducting sandwich cylinder with a planar lattice core under prescribed eigenstrain and magnetic field. Composite Structures, 2016. 153: p. 632-644.

14. Lu, Z., et al., Elastic properties of two novel auxetic $3 D$ cellular structures. International Journal of Solids and Structures, 2017. 124: p. $46-56$.

15. Courant, R. and D. Hilbert, Methods of Mathematical Physics. 2008: Wiley-VCH Verlag GmbH. I-XXII.

16. Singh, R., Thermal Conduction Through Porous Systems, in Cellular and Porous Materials. 2008, Wiley-VCH Verlag GmbH \& Co. KGaA. p. 199-238.

17. Bauer, T.H., A general analytical approach toward the thermal conductivity of porous media. International Journal of Heat and Mass Transfer, 1993. 36: p. 4181-4191.

18. Baillis, D. and R. Coquard, Radiative and Conductive Thermal Properties of Foams, in Cellular and Porous Materials. 2008, Wiley-VCH Verlag GmbH \& Co. KGaA. p. 343-384.

19. Gong, L., et al., A novel effective medium theory for modelling the thermal conductivity of porous materials. International Journal of Heat and Mass Transfer, 2014. 68: p. 295-298.
20. Hashin, Z. and S. Shtrikman, A Variational Approach to the Theory of the Effective Magnetic Permeability of Multiphase Materials. Journal of Applied Physics, 1962. 33(10): p. 3125-3131.

21. Fiedler, T., et al., Optimized Lattice Monte Carlo for thermal analysis of composites. Computational Materials Science, 2014. 95: p. 207-212.

22. Karttunen, A.T., J.N. Reddy, and J. Romanoff, Micropolar modeling approach for periodic sandwich beams. Composite Structures, 2018. 185: p. 656-664.

23. Hollister, S.J. and N. Kikuchi, A comparison of homogenization and standard mechanics analyses for periodic porous composites. Computational Mechanics, 1992. 10(2): p. 73-95.

24. S Bakhvalov, N. and G. Panasenko, Homogenisation: Averaging processes in periodic media. . 1989.

25. Incropera, F.P., Fundamentals of Heat and Mass Transfer. 2006: John Wiley $\backslash \&$ Sons.

26. Matsuura, M., Gielis' superformula and regular polygons. Journal of Geometry, 2015. 106(2): p. 383-403.

27. Mackay, A.L., Periodic minimal surfaces. Nature, 1985. 314: p. 604.

28. Deng, Y. and M. Mieczkowski, Three-dimensional periodic cubic membrane structure in the mitochondria of amoebaeChaos carolinensis. Protoplasma, 1998. 203(1): p. 16-25.

29. Gan, Z., M.D. Turner, and M. Gu, Biomimetic gyroid nanostructures exceeding their natural origins. Science Advances, 2016. 2.

30. Shin, J., et al., Finite Element Analysis of Schwarz P Surface Pore Geometries for Tissue-Engineered Scaffolds. Vol. 2012. 2012.

31. Kapfer, S.C., et al., Minimal surface scaffold designs for tissue engineering. Biomaterials, 2011. 32(29): p. 6875-6882.

32. Lei, J. and Z. Liu, The structural and mechanical properties of graphene aerogels based on Schwarz-surface-like graphene models. Carbon, 2018. 130: p. 741-748.

33. Maskery, I., et al., Insights into the mechanical properties of several triply periodic minimal surface lattice structures made by polymer additive manufacturing. Polymer, 2017.

34. Collishaw, P.G. and J.R.G. Evans, an Assessment of Expressions for the Apparent Thermal-Conductivity of Cellular Materials. Journal of Materials Science, 1994. 29(9): p. 2261-2273.

35. Fiedler, T., I.V. Belova, and G.E. Murch, Theoretical and Lattice Monte Carlo analyses on thermal conduction in cellular metals. Computational Materials Science, 2010. 50(2): p. 503-509.

36. Boldrin, L., F. Scarpa, and R. Rajasekaran, Thermal conductivities of iso-volume centre-symmetric honeycombs. Composite Structures, 2014. 113(1): p. 498-506.

37. Van De Walle, W. and H. Janssen, Validation of a $3 D$ pore scale prediction model for the thermal conductivity of porous building materials. Energy Procedia, 2017. 132: p. 225-230.

38. Abueidda, D.W., et al., Effective conductivities and elastic moduli of novel foams with triply periodic minimal surfaces. Mechanics of Materials, 2016. 95: p. 102-115. 